Molecular

Syndromology
Editorial - Late Breaking Chromosomes

Mol Syndromol 2021;12:65-68

DOI: $10.1159 / 000515363$

\title{
Precision Medicine: from Molecular Diagnoses to Treatment Opportunities in Medical Genetics
}

Using cytogenetic and molecular genetic techniques, medical genetics aims to uncover the structural or molecular abnormalities that may underlie the clinical conditions of the patients under study. These investigations serve purposes such as understanding the biology of the disorders and their recurrence risk within the affected family. Clinical medicine goes beyond diagnosis by providing treatment options. Recently, the latter has become within reach for genetic disorders as well. Under terms such as "precision medicine," individualized treatments guided by molecular genetic diagnosis have been devised for numerous inherited disorders, including several forms of refractory epilepsy.

In a recent issue of Molecular Syndromology, an example of such precision medicine, i.e., the successful treatment of a female patient with speech delay, tonicclonic seizures, and a heterozygous de novo mutation in the NEXMIF gene was published [Ogasawara et al., 2020]. Loss of NEXMIF provokes a dysregulation in synaptic protein expression and a reduction in both glutamatergic and GABAergic synaptic proteins [Gilbert et al., 2020]. Reduced GABA signaling is implicated in some forms of epilepsy [Oakley et al., 2013]. The GABA-enhancing drug clonazepam likely ameliorates epilepsy due to the decreased GABAergic synaptic activity resulting from the loss-of-function of NEXMIF [Oakley et al., 2013]. This is the third successful treatment of an epilepsy patient with a NEXMIF gene mutation published. Case reports like this point us toward novel treatment avenues for patients with genetic forms of epilepsy.

karger@karger.com

(c) 2021 S. Karger AG, Basel

www.karger.com/msy

Karger"
A second group of epilepsy cases with a clear rationale for treatment are patients with dominant gain-offunction mutations in genes encoding the members of the N-methyl-D-aspartate receptors (NMDARs), i.e., GRIN2A, GRIN2B, and GRIN2D. NMDARs are ligandgated ionotropic receptors mediating a slow, calciumpermeable component of the excitatory synapses in the central nervous system. They are composed of di- or triheterotetrameric ligand-gated ion channels consisting of 2 glycine-binding GluN1 (encoded by GRIN1) and 2 glutamate-binding GluN2 subunits (encoded by the GRIN2A - GRIN2D genes) [Traynelis et al., 2010]. Thus far, GRIN1, GRIN2A, GRIN2B, and GRIN2D have been linked to human disease [Carvill et al., 2013; Lemke et al., 2013, Lesca et al., 2013, Hu et al., 2016, Fry et al., 2018].

GRIN2D encodes the GluN2D subunit protein of the NMDAR, which is mutated in childhood epilepsy disorders such as West syndrome (MIM: 308350) and Landau Kleffner syndrome (MIM: 245570). A de novo recurrent missense variant, c.1999G > A (p.Val667Ile), in GRIN2D was identified by exome and panel sequencing in 2 unrelated children with epileptic encephalopathy [Li et al., 2016]. Due to this variant, a valine in the third transmembrane domain of the GluN2D protein was replaced by an isoleucine. This change altered the pharmocologic properties of the NMDAR in such a way that responses to either glutamate and glycine were increased, the sensitivity to negative allosteric modulators was reduced, the response time course to synaptic-like stimulation was pro-

Correspondence to:

Martin Poot, martin_poo@@hotmail.com 
longed, and the probability that an agonist-bound single channel will open was increased 6-fold.

The 2 patients with this gain-of-function variant did not respond to conventional antiepileptic treatments. FDA-approved NMDAR antagonists, including memantine, ketamine, amantadine, and dextromethorphan were thus evaluated. Of these, memantine elicited the strongest effect. Patient 1 showed subjective developmental gains but no seizure improvement [XiangWei et al., 2019]. After the patient's seizures worsened and developed to refractory status epilepticus, these were controlled by combination therapy with ketamine and magnesium sulfate. Patient 2 responded initially to memantine treatment with seizure control and some developmental improvement, although she had no speech and was not able to walk. Later, she had refractory epilepsy with 5-7 (mostly tonic) seizures per day, controlled with a combination of memantine, lamotrigine, and valproic acid. A third, new patient, with the same variant, became completely free of seizures during treatment with memantine, intravenous immunoglobulins, oral steroids, and magnesium [XiangWei et al., 2019]. While these findings are encouraging, the variablity in response to memantine treatment of the 3 patients with the same variant clearly needs further study.

Platzer et al. [2017] studied a cohort of 86 patients with probable or likely pathogenic GRIN2B variants and neurodevelopmental disorders including intellectual disability, epilepsy, and autism, and associated with a spectrum of hypotonia, movement disorder, cortical visual impairment, cerebral volume loss, and epilepsy. In 6 patients, a consistent pattern of malformation of cortical development was observed. The 86 likely pathogenic variants comprised 52 distinct missense variants. With one exception, they all clustered within, or in close proximity to, the ligand-binding domains S1 and S2 as well as the transmembrane domains M1-M4. Of these, 21 distinct pathogenic variants lead presumably to protein truncation or haploinsufficiency. Interestingly, the patients with protein-truncating variants tended to present with mild or moderate instead of severe intellectual delay. In addition, there were 6 probable gain-of-function variants. These were selected for treatment with memantine to reduce NMDAR hyperactivity. Initially observed beneficial effects such as improvements in awareness, behavior, and sleep could not be sufficiently corroborated later on. In addition, there were no changes in seizure frequency.

In a single patient with a de novo missense mutation in GRIN2A (c.2434C > A; p.L812M), that provoked an elevated charge transfer in the NMDAR, memantine reduced the proband's seizure burden [Pierson et al., 2014].
In a cohort of 248 patients with pathogenic or likely pathogenic variants in GRIN2A, the phenotypic spectrum ranged from normal or near-normal development, with mild epilepsy and speech delay or apraxia, to severe developmental and epileptic encephalopathy, sometimes within the epilepsy-aphasia spectrum [Strehlow et al., 2019]. Pathogenic missense variants in the transmembrane and linker domains of the encoded protein were associated with more severe developmental phenotypes, whereas missense variants within amino terminal or ligand-binding domains and null variants provoked less severe phenotypes. These 2 groups also differ with respect to phenotypes such as MRI abnormalities and epilepsy types. These findings were congruent with electrophysiology data. Variants in the transmembrane and linker domains were predominantly associated with NMDAR gain-of-function, while those in the amino terminal or ligand-binding domains caused NMDAR loss-of-function only. Null variants caused a reduced NMDAR function, without any evidence for compensatory overexpression of GluN2B. The authors state that this pathomechanistic model may help in predicting phenotype severity as well as eligibility for potential precision medicine approaches in GRIN2A-related disorders.

In a study of cultured cells transfected with 4 missense variants of GRIN2A, a reduction in the response to glutamate and glycine was observed, which could be rescued by adding a GluN2A-selective positive allosteric modulator of the thiazolopyrimidinone-family of drugs [Addis et al., 2017].

Apart from the NMDAR-related epilepsies, successful treatment of several other patients after molecular diagnosis have been reported. From a cohort of 36 patients with SNCA8-related epilepsies, 21 responded to treatment with established antiepileptic drugs, including carbamazepine, clobazam, ethosuximide, levetiracetam, lamotrigine, oxcarbazepine, phenobarbital, perampanel, phenytoin, topiramate, vigabatrin, and valproate [Johannesen et al., 2019]. The extent and the kind of response (positive or adverse) varied across patients and did not reflect a preponderance for any of the affected protein domains.

A girl with frequent myoclonic and apneic seizures during the neonatal period (early myoclonic encephalopathy) and a heterozygous de novo missense mutation in the SCN1A gene (c.2588 T > C:p.Leu863Ser) responded well to a combination of potassium bromide, phenobarbital, clobazam, and perampanel, after phenytoin (followed by phenobarbital) provided transient effects and topiramate, clonazepam, potassium bromide, and cloba-
66

Mol Syndromol 2021;12:65-68 DOI: $10.1159 / 000515363$ 
zam (administered serially) exerted little effect [Ishikawa et al., 2019]. Perampanel is a noncompetitive $\alpha$-amino-3hydroxyl-5-methyl-4-isoxazole-propionate (AMPA) receptor antagonist. The AMPA receptor is the main excitatory postsynaptic glutamate receptor. The authors indicated that further studies are needed to confirm the efficacy of perampanel treatment against early myoclonic epilepsy.

Lennox Gastaut syndrome is a severe childhood epilepsy disorder which may, among other variants, be caused by mutations in the $\beta 3$ subunit of the $\mathrm{GABA}_{\mathrm{A}}$ receptor [Allen et al., 2013]. This subunit governs the assembly and trafficking of the receptor to the cell surface [Connolly et al., 1996]. Several variants in the N-terminal extracellular random coil close to the first transmembrane (M1) segment are associated with Lennox Gastaut syndrome [Shi et al., 2019]. Seizures in patients with this disorder are generally refractory to valporate and lamotrigine [Shi et al., 2019]. In a recent study, a patient with a c.905A $>$ G variant in the GABRB3 gene that caused a $Y 302 \mathrm{C}$ change in the amino acid sequence of the protein was administered a synthetic vinca alkaloid (vinpocetine) as a dietary supplement [Billakota et al., 2019]. This treatment resulted in a sustained, dose-dependent reduction in spike-wave discharge frequency on electroencephalogram and improved the patient's language and behavior. In view of the severity and treatment resistance of Lennox Gastaut syndrome, vinpocetine merits further consideration.

In a cohort of 1,019 patients with various forms of epilepsy, together with significant developmental comor- bidities, 139 individuals carried a possibly or definitely pathogenic copy number variation [Coppola et al., 2019]. Of these, 5 affected the HNRNPU gene. This gene has initially been pinpointed by deletion mapping of 4 patients with epilepsy, neurodevelopmental comorbidities, and a chromosomal loss in the 1q44 region [Caliebe et al., 2010]. The pathological significance of $H N R N P U$ for epileptic encephalopathy has been corroborated by studies of single nucleotide variants [Bramswig et al., 2017; Depienne et al., 2017; Leduc et al., 2017]. Thus far, no treatments have been reported for these patients.

A patient with early infantile epileptic encephalopathy (EIEE) type 54 (caused by a heterozygous HNRNPU variant), severe hyperventilation, and apnea was treated with a combination of acetazolamide, alprazolam, and aripiprazole, as reported in this issue of Molecular Syndromology [Spagnoli et al., 2021]. This the first patient with a HNRNPU variant and breathing abnormalities for which pharmacological treatment has been reported.

Thus far, individualized treatment guided by molecular genetic diagnosis has been offered to only a few patients, but the number of diseases and, consequently, the number of patients eligible for precision medicine is rapidly growing, as is reflected by the surge of reports in the past few years (see above). Molecular Syndromology aims to support this emerging development on the intersection between medical genetics and clinical medicine with an article series on "Molecular Genetic Diagnosis-based Precision Medicine." All readers and authors are cordially invited to participate in this article series by submitting papers on this topic.

Martin Poot

\section{References}

Addis L, Virdee JK, Vidler LR, Collier DA, Pal DK, Ursu D. Epilepsy-associated GRIN2A mutations reduce NMDA receptor trafficking and agonist potency - molecular profiling and functional rescue. Sci Rep. 2017 Feb;7(1):66.

Allen AS, Berkovic SF, Cossette P, Delanty N, Dlugos D, Eichler EE, et al.; Epilepsy Phenome/Genome Project. De novo mutations in epileptic encephalopathies. Nature. 2013 Sep; 501(7466):217-21.

Billakota S, Andresen JM, Gay BC, Stewart GR, Fedorov NB, Gerlach AC, et al. Personalized medicine: vinpocetine to reverse effects of GABRB3 mutation. Epilepsia. 2019 Dec; 60(12):2459-65.

Bramswig NC, Lüdecke HJ, Hamdan FF, Altmüller J, Beleggia F, Elcioglu NH, et al. Heterozygous HNRNPU variants cause early onset epilepsy and severe intellectual disability. Hum Genet. 2017 Jul;136(7):821-34.
Caliebe A, Kroes HY, van der Smagt JJ, MartinSubero JI, Tönnies H, van 't Slot R, et al. Four patients with speech delay, seizures and variable corpus callosum thickness sharing a $0.440 \mathrm{Mb}$ deletion in region 1q44 containing the HNRPU gene. Eur J Med Genet. 2010 JulAug;53(4):179-85.

Carvill GL, Regan BM, Yendle SC, O'Roak BJ, Lozovaya N, Bruneau N, et al. GRIN2A mutations cause epilepsy-aphasia spectrum disorders. Nat Genet. 2013 Sep;45(9):1073-6.

Connolly CN, Krishek BJ, McDonald BJ, Smart TG, Moss SJ. Assembly and cell surface expression of heteromeric and homomeric gamma-aminobutyric acid type A receptors. J Biol Chem. 1996 Jan;271(1):89-96.

Coppola A, Cellini E, Stamberger H, Saarentaus E, Cetica V, Lal D, et al.; EuroEPINOMICSRES Consortium; EpiCNV Consortium. Diagnostic implications of genetic copy number variation in epilepsy plus. Epilepsia. 2019 Apr;60(4):689-706.

Depienne C, Nava C, Keren B, Heide S, Rastetter A, Passemard S, et al.; DDD Study. Genetic and phenotypic dissection of 1q43q44 microdeletion syndrome and neurodevelopmental phenotypes associated with mutations in ZBTB18 and HNRNPU. Hum Genet. 2017 Apr;136(4):463-79.

Fry AE, Fawcett KA, Zelnik N, Yuan H, Thompson BA, Shemer-Meiri L, et al. De novo mutations in GRIN1 cause extensive bilateral polymicrogyria. Brain. 2018 Mar;141(3):698712.

Gilbert J, O'Connor M, Templet S, Moghaddam M, Di Via Ioschpe A, Sinclair A, et al. NEXMIF/KIDLIA knock out mouse demonstrates autism-like behaviors, memory deficits, and impairments in synapse formation and function. J Neurosci. 2020 Jan;40(1):237-54.
Precision Medicine: from Molecular Diagnoses to Treatment Opportunities
Mol Syndromol 2021;12:65-68 DOI: $10.1159 / 000515363$ 
Hu C, Chen W, Myers SJ, Yuan H, Traynelis SF. Human GRIN2B variants in neurodevelopmental disorders. J Pharmacol Sci. 2016 Oct; 132(2):115-21.

Ishikawa N, Tateishi Y, Tani H, Kobayashi Y, Itai T, Miyatake S, et al. Successful treatment of intractable life-threatening seizures with perampanel in the first case of early myoclonic encephalopathy with a novel de novo SCN1A mutation. Seizure. 2019 Oct;71:20-3.

Johannesen KM, Gardella E, Encinas AC, Lehesjoki AE, Linnankivi T, Petersen MB, et al. The spectrum of intermediate SCN8A-related epilepsy. Epilepsia. 2019 May;60(5):830-44.

Leduc MS, Chao HT, Qu C, Walkiewicz M, Xiao $\mathrm{R}$, Magoulas $\mathrm{P}$, et al. Clinical and molecular characterization of de novo loss of function variants in HNRNPU. Am J Med Genet A. 2017 Oct;173(10):2680-9.

Lemke JR, Lal D, Reinthaler EM, Steiner I, Nothnagel M, Alber M, et al. Mutations in GRIN2A cause idiopathic focal epilepsy with rolandic spikes. Nat Genet. 2013 Sep;45(9):1067-72.

Lesca G, Rudolf G, Bruneau N, Lozovaya N, Labalme A, Boutry-Kryza N, et al. GRIN2A mutations in acquired epileptic aphasia and related childhood focal epilepsies and encephalopathies with speech and language dysfunction. Nat Genet. 2013 Sep;45(9):1061-6.
Li D, Yuan H, Ortiz-Gonzalez XR, Marsh ED, Tian L, McCormick EM, et al. GRIN2D Recurrent De Novo Dominant Mutation Causes a Severe Epileptic Encephalopathy Treatable with NMDA Receptor Channel Blockers. Am J Hum Genet. 2016 Oct;99(4):802-16.

Oakley JC, Cho AR, Cheah CS, Scheuer T, Catterall WA. Synergistic GABA-enhancing therapy against seizures in a mouse model of Dravet syndrome. J Pharmacol Exp Ther. 2013 May;345(2):215-24.

Ogasawara M, Nakagawa E, Takeshita E, Hamanaka K, Miyatake S, Matsumoto N, et al. Clonazepam as an Effective Treatment for Epilepsy in a Female Patient with NEXMIF Mutation: case Report. Mol Syndromol. 2020 Nov;11(4):232-7.

Pierson TM, Yuan H, Marsh ED, Fuentes-Fajardo K, Adams DR, Markello T, et al.; PhD for the NISC Comparative Sequencing Program. GRIN2A mutation and early-onset epileptic encephalopathy: personalized therapy with memantine. Ann Clin Transl Neurol. 2014 Mar;1(3):190-8.

Platzer K, Yuan H, Schütz H, Winschel A, Chen W, Hu C, et al. GRIN2B encephalopathy: novel findings on phenotype, variant clustering, functional consequences and treatment aspects. J Med Genet. 2017 Jul;54(7):460-70.
Shi YW, Zhang Q, Cai K, Poliquin S, Shen W, Winters N, et al. Synaptic clustering differences due to different GABRB3 mutations cause variable epilepsy syndromes. Brain. 2019 Oct;142(10):3028-44.

Spagnoli C, Rizzi S, Salerno GG, Frattini D, Koskenvuo J, Fusco C. Pharmacological treatment of severe breathing abnormalities in a case of HNRNPU epileptic encephalopathy. Mol Syndromol. 2021. DOI: $10.1159 / 000512566$.

Strehlow V, Heyne HO, Vlaskamp DR, Marwick KF, Rudolf G, de Bellescize J, et al.; GRIN2A study group. GRIN2A-related disorders: genotype and functional consequence predict phenotype. Brain. 2019 Jan;142(1):80-92.

Traynelis SF, Wollmuth LP, McBain CJ, Menniti FS, Vance KM, Ogden KK, et al. Glutamate receptor ion channels: structure, regulation, and function. Pharmacol Rev. 2010 Sep;62(3): 405-96.

XiangWei W, Kannan V, Xu Y, Kosobucki GJ, Schulien AJ, Kusumoto H, et al. Heterogeneous clinical and functional features of GRIN2D-related developmental and epileptic encephalopathy. Brain. 2019 Oct;142(10): 3009-27. 